\title{
"Acque calde" ai congressi di Mórahalom (Ungheria) e a Idrovulc2013
}

\author{
Daniela Ducci - Segretario del Comitato Italiano IAH - daniela@unina.it \\ Marco Petitta - Presidente del Comitato Italiano IAH - marco.petitta@uniroma1.it
}

La comunità idrogeologica nazionale e internazionale ha sempre riservato un ruolo da protagonista alle risorse idriche degli acquiferi vulcanici, sia per l'entità di tale risorsa, che per le peculiarità delle acque erogate da questi acquiferi, utilizzate sia a scopo minerale che termale. Negli ultimi anni, si è aggiunto l'interesse verso le risorse geotermiche, anche laddove non siano legate a manifestazioni vulcaniche. Lo scorso mese di maggio due diverse iniziative hanno affrontato queste problematiche con la partecipazione attiva della IAH: la Conferenza dell'Europa Centrale sulle acque sotterranee, intitolata proprio alle risorse geotermiche, tenutasi nell'Ungheria Meridionale a cura del Comitato IAH ungherese, e il convegno nazionale IdroVulc2013 "Acquiferi Vulcanici dell'Italia centrale: Studi idrogeologici per la soluzione di problemi gestionali", organizzato dall'Università degli Studi Roma TRE e dalla Regione Umbria.

Dal 6 all'8 maggio nella cittadina di Mórahalom, vicino al confine con Serbia ed Ungheria, ha avuto luogo la conferenza dedicata alle applicazioni in campo geotermico delle risorse idriche sotterranee. In un ambiente amichevole e stimolante dal punto di vista scientifico, i colleghi ungheresi hanno organizzato una conferenza di ottimo livello scientifico, con la partecipazione di circa 90 studiosi e ricercatori provenienti da molti paesi europei; la delegazione italiana forte, di 6 membri, è risultata la più numerosa dopo i padroni di casa.

Le sessioni scientifiche hanno spaziato dalle questioni tecniche su perforazioni e gestione della risorsa agli studi e strumenti per valutare l'origine delle acque termali, nonché dagli aspetti normativi fino alle tecniche di modellazione del flusso e del calore nei serbatoi idrici sotterranei. Lelevato livello scientifico è stato impreziosito dalla partecipazione attiva di esperti di fama internazionale quali József Tóth, dell'University of Alberta in Canada, capostipite della scuola ungherese di idrogeologia e László Rybach, altro illustre ungherese trapiantato all'ETH di Zurigo. Il taglio della conferenza, con un numero limitato di partecipanti, ha favorito la discussione, lo scambio di esperienze e risultati, consentendo inoltre possibili future collaborazioni tra diversi paesi, nello spirito che la Commissione Europea vuole dare alla ricerca scientifica anche in campo idrogeologico, verso una sempre più stretta internazionalizzazione degli studi sia di campo che di laboratorio. Gli aspetti conviviali e il field-trip finale hanno aumentato le occasioni di incontro in un clima molto accogliente. In definitiva, a detta di tutti i partecipanti italiani, la conferenza si è rivelata un ottimo momento di confronto con i paesi dell'Europa Centrale ed Orientale, con i quali non sono molto frequenti le collaborazioni in campo idrogeologico. C'è quindi la speranza fondata che si aprano nuovi orizzonti per la comunità scientifica e, in prospettiva, anche per i professionisti.

Il 16 e il 17 maggio si è tenuto ad Orvieto, nella splendida cornice del rinascimentale Palazzo del Popolo, il Convegno IdroVulc2013 “Acquiferi Vulcanici dell'Italia centrale: Studi idrogeologici per la soluzione di problemi gestionali” (Fig. 1).

Il Convegno era focalizzato sugli acquiferi vulcanici del settore periterrenico dell'Italia centrale, che costituiscono un'importante risorsa per l'approvvigionamento idrico locale, talora con notevoli problemi gestionali legati alle peculiarità qualitative delle acque sotterranee nonché al sovrasfruttamento della risorsa. Per superare tali problemi, Il Convegno si è posto come obiettivo l'apertura di una discussione fra la comunità scientifica e accademica, gli Enti gestori della risorsa idrica, le Amministrazioni e i professionisti locali, mirata alla redazione sinergica di norme tecniche e strumenti gestionali e pianificatori a varie scale.

Il pomeriggio del 16, dopo la presentazione del Convegno da parte del prof. Roberto Mazza e la conferenza stampa che ha visto la partecipazione dei politici locali, particolarmente impegnati nel campo ambientale, si è tenuta la sessione poster e l'ice-breaking aperitif, che ha consentito ai partecipanti di familiarizzare e ha reso particolarmente piacevole l'atmosfera congressuale. Tale atmosfera è poi proseguita nella cena, presso il ristorante "al S. Francesco" che ha visto un piacevole e stimolante merge tra i vari ambienti presenti al Convegno (scientifico, amministrativo e professionale), oltre ad accattivanti aspetti culinari...

Il giorno 17, dopo i saluti delle Autorità e gli interventi degli Assessori all'Ambiente della Regione Umbria e del Comune di Orvieto e dei rappresentanti degli Ordini regionali dei geologi (Umbria, Toscana, Lazio e Campania) si sono svolte quattro sessioni inerenti i seguenti argomenti:

- Gestione degli acquiferi con scadenti caratteristiche qualitative della risorsa

- Gestione degli acquiferi interessati da prelievo di acque minerali e termali

- Gestione degli acquiferi in condizioni di sovrasfruttamento della risorsa.

- Gestione degli acquiferi interessati dalla produzione di energia geotermica a bassa entalpia

Per tutte le sessioni, presiedute da due chairmen, uno del mondo accademico e uno del mondo tecnico-amministrativo, si è avuto un intervento ad invito, seguito da 3 comunicazioni sull'argomento. Sulle 4 sessioni si sono inoltre avuti più di 20 contributi poster.

Nella prima sessione gli argomenti più frequenti sono stati senza dubbio la contaminazione da Arsenico e i "Valori di 
fondo", sensu D.Lgs. 30/2009. Molti lavori presentati hanno inoltre illustrato metodologie innovative, avvalendosi in particolar modo di analisi statistiche (frequenza, trends, etc.), con applicazioni a casi di studio, relativi prevalentemente a Campania, Lazio e Toscana.

Nella seconda sessione sono stati illustrati casi di studio relativi a importanti aree minerali e termo-minerali del centrosud Italia, in termini di caratterizzazione idrochimica e rappresentazioni cartografiche.

La terza sessione ha analizzato gli aspetti quantitativi degli acquiferi vulcanici, attraverso metodologie di valutazione delle aliquote delle componenti del ciclo idrologico, con particolare riguardo all'impatto dei prelievi di acque sotterranee sulle falde. Molti lavori si sono basati sull'uso di modelli numerici, finalizzati anche ad individuare i fattori critici per la gestione sostenibile delle risorse. Anche in questa sessione l'aspetto metodologico ha trovato sempre applicazione in interessanti casi di studio.
La quarta sessione ha infine consentito un quadro completo e variegato dello stato dell'arte della produzione di energia geotermica a bassa entalpia nelle regioni peritirreniche dell'Italia centrale e sulle implicazioni idrogeologiche. Approfonditi interventi hanno analizzato i vantaggi e gli svantaggi dei sistemi a circuito aperto e a circuito chiuso, per la circolazione idrica sotterranea e per le caratteristiche chimiche e termiche delle acque sotterranee.

Tutti gli interventi sono stati seguiti da ampi ed accesi dibattiti, volti ad approfondire le comunicazioni e ad avviare confronti costruttivi tra esperienze diversificate. E tra questi si segnala la tavola rotonda finale che ha dimostrato come il Convegno, che ha contato più di 250 iscritti, abbia perfettamente centrato l'obiettivo, stimolando i vari soggetti interessati (comunità scientifica, enti, amministrazioni locali e mondo professionale) a trovare strategie comuni e risolutive per la gestione ottimale della risorsa.

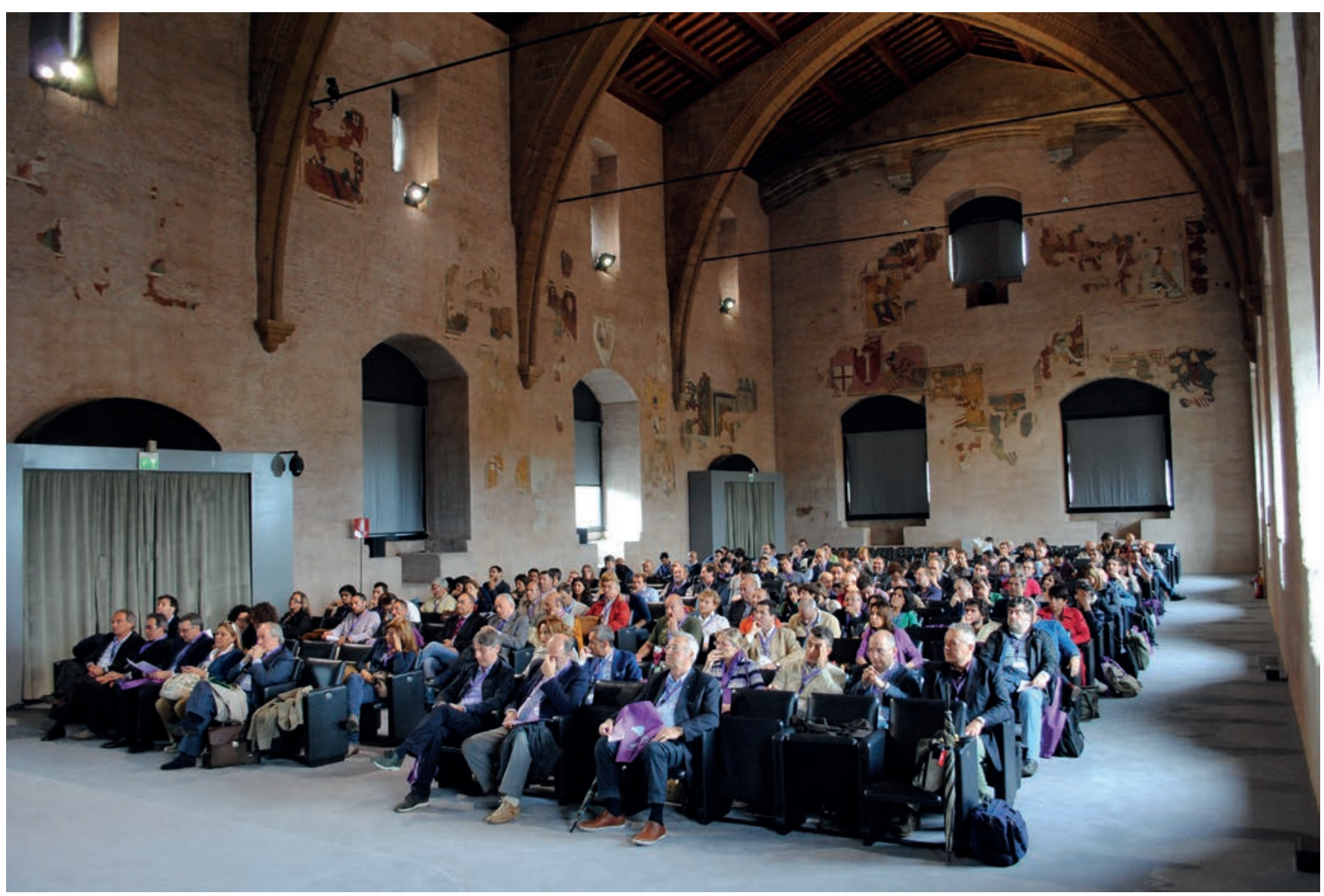

Fig. 1 - La "sala dei Quattrocento" del Palazzo del Popolo (Orvieto), affollata dai partecipanti al convegno Idrovulc (foto di Stefano Viaroli). 